\title{
Effects of taping application type on grip power, pinch power, and EMG activity
}

\author{
Young-Han Park
}

Department of Physical Therapy, Korea National University of Transportation, Jeungpyeong-gun, South Korea

\section{Email address:}

yhpark@ut.ac.kr

\section{To cite this article:}

Young-Han Park. Effects of Taping Application Type on Grip Power, Pinch Power, and EMG Activity. Science Journal of Public Health. Vol. 1, No. 5, 2013, pp. 239-243. doi: 10.11648/j.sjph.20130105.20

\begin{abstract}
This study is to investigate the changes of grip power, pinch power, EMG (MVC) and to generalize the effects by differentiating the application of tape on hand which we use the most in daily living. All subjects were divided into three groups, FT, ET, and RT group. All groups conducted pre-test and grip/pinch power and EMG were measured before and after the application of taping. Hand dynamometer was used to measure the grip power of hand. Measuring instrument for pinch power was used to investigate the pinch power according to the strength of hand. Maximum grip force and forearm surface EMG were recorded for maximum voluntary isometric grip contractions. After the application of flexor taping, there was a significant change in grip power $(p<0.05)$, but not significant in pinch power $(p>0.05)$. After the application of extensor taping, both grip power and pinch power showed some increases, but no significance ( $p>0.05)$. After the application of ring taping, there was a significant change $(p<0.05)$, but not statistically significant in the pinch power $(p>0.05)$. Application of ring taping was significant increase at the MVC compared with other groups ( $<<0.05)$. Flexor and ring type of taping therapy increased the grip power, MVC of hand and is expected to be used as a auxiliary tool for improvement of strength and function in patients who have low hand function because of frequent use of hands.
\end{abstract}

Keywords: Taping, Grip Power, Pinch Power, EMG

\section{Introduction}

All of physical activities are based on the muscular contraction. Muscle strength, muscle power and muscle endurance are essential components for the performance of athletes $[1,2]$. Muscle strength is defined as the ability of the muscle to generate the maximal contractile force against the resistance. Thus, muscle strength is rated as the most important component to decide the exercise ability [3] Muscle strength can increase the working volume and decrease the poor position and the injury resulted from the lack of exercise. Furthermore, it can also improve the motor ability and protect the injury caused in emergency [4, $5]$.

Hand is the important part of body to perform the complex daily living, although it is small of many parts of body [6]. Most of daily activity depends on the proper function and it can be easily injured because of its characteristics such as complexity of structure and exposed part. Especially, the physical activity of human beings was decreased in general as modern society got mechanized and industrialized, resulting in the overall decrease of motor ability. In addition, the function of hand gets more important as the work in sitting position becomes more frequent than in standing position and the more diseases related to hands occurred because of the repetitive stress and injuries. Effective interventions which are easily applicable and affordable with economy of time, without location restriction are needed [7].

Taping therapy is recently used for pain relief and enhancement of performance in many sport organization and hospitals, which is noninvasive, user-friendly, and consistently analgesic effective as a field of complementary medicine [8].

Elastic tape was developed for the treatment of patients in 1985, then, it is used not only for treatments but also for sport plays. After 'Kinesio', called the functional tape, was introduced in Korea in 1991, it has been often shown that players used the elastic tape in all sorts of sports. It was to protect the injury during the game and to control the elaborate muscle activities. Furthermore, taping is being used to relieve the pain and to improve the performance 
and exercise ability $[9,10]$.

Elastic taping facilitates or restricts the muscle tension by applying the tape to the skin on relevant muscles to normalize the action of agonist in moving joint. This tape is widely used for function strengthening and local pain relief through the effect of physiological reflection such as compression, traction and sagging according to the mechanical stimulation [11]. The pain relief has been attempted by directly attaching tapes on skin and muscle in musculoskeletal system injury $[12,13]$ and many ways of taping have been developed, applied and studied for purpose of improvement in muscle strength and muscle endurance [14].

Of many studies that elastic taping improves the function of muscles, Choi and Kim [15] reported that the way of attaching tapes on joints and muscles to maximize the performance ability improves the function of muscle and joint, as well as protect the injury. Furthermore, Singletary and Geissler [16] reported that the improvement of muscle strength decreased the risks of re-fracture in fracture patients with taping. Some study reported the application of kinesio tape on muscles related to swing motion of golfer resulted in muscle strengthening and maintenance of exercise ability and Jung and Lee also reported that lumbar region taping is highly useful for the assistance of muscle strengthening because it has a great impact on muscle recruitment [17].

However, most of researches that have been studied were about the lower extremity and there are few studies about hand which is used the most in daily living. In addition, previous studies have focused on tape's own effect about pain relief or muscle strengthening, but there is a lack of investigation about methodological effects for the correct treatment. Thus, this study is to investigate the changes of grip/pinch power, EMG and to generalize the effects by differentiating the application of tape on hand which we use the most in daily living.

\section{Method}

\subsection{Subjects}

45 normal students of $\mathrm{T}$ university in Jeungpyeong were recruited to investigate changes of the grip/pinch power and EMG by applying the taping treatment. All subjects were divided into three groups, FT, ET, and RT group. All groups conducted pre-test and grip/pinch power and EMG were measured before and after the application of taping.

The exclusion criteria is as following; 1) pain or motor disturbance around hand or arm, 2) pressure pain 3) medication, 4) burn on measuring part. All experiments were conducted with patients' consents after information about this study like procedure and purpose had been given.

\subsection{Experimental Equipment and Procedure}

Kinesio tape (Kinesio Tex Gold, KINESIO, JAPAN), elastic tape, was used in this study. It was attached respectively on flexor, extensor of hand and wrist in bracelet form by one therapist according to three groups. For adaptation of subjects to the test and treatment, the test was conducted after pre-application period of 2 days.

Hand dynamometer (Hydraulic Hand Dynamometer, JAMAR, USA) was used to measure the grip power of hand. To improve the maximal strength and objectivity, the subjects had to maintain the posture with sitting on the chair, forearm on trunk, shoulder on neutral position, and elbow flexed by 90 degrees during measurement. The grip test was conducted for 5 seconds without any pain and there were 5 minute-breaks between tests to sufficiently recover the fatigue. Measurements were performed total 6 times, respectively 3 times before and after the application of taping. Then, the mean value was used for statistical analysis.

Measuring instrument for pinch power (Baseline Hydraulic Pinch Grage, JAMAR, USA) was used to investigate the pinch power according to the strength of hand, and the measurements were performed in the same way of grip power. The tests were conducted in stable condition in the afternoon after daily work, considering subjects' conditions.

Surface electromyography of three muscles on the forearm; flexor carpi ulnaris (FCU), flexor carpi radialis (FCR), palmaris longus (PL), were recorded. Each electrode pair was positioned on the midline of the muscle belly at the mid-point between the myotendinous junction and the muscle belly. A TeleMyo 2400T-G2 16 channel telemetric SEMG system (Noraxon, USA, 2007) with associated software MyoResearch XP Master Edition (Noraxon, USA) was used to collect, process, and analyze the EMG signals of the muscles. Maximum grip force and forearm surface EMG were recorded for maximum voluntary isometric grip contractions (MVC).

\subsection{Statistical Analysis}

SPSS 18.0 for window was used for statistical analysis in this study. Descriptive statistic was performed to investigate the general characteristics of all subjects and data was described as mean \pm SD. Paired T-test was also used to analyze the effect of taping treatment on grip and pinch power in each group. For inter-group comparison, one-way ANOVA was used. Significance level was set as $\mathrm{p}<0.05$.

\section{Result}

\subsection{General Characteristics of Subjects}

General characteristics of subjects were as following; 1) age of $20.26 \pm 2.29$ year, height of $166.15 \pm 9.05 \mathrm{~cm}$, weight of $59.21 \pm 13.1 \mathrm{~kg}$ in FT group. 2) age of $20.25 \pm 1.85$ year, height of $164.84 \pm 8.18 \mathrm{~cm}$, weight of $58.90 \pm 15.20 \mathrm{~kg}$ in ET group. 3) age of $20.64 \pm 1.94$ year, height of $165.60 \pm 6.96 \mathrm{~cm}$, weight of $56.90 \pm 9.27<$ Table $1>$. 
Table 1. General characteristics of subjects

\begin{tabular}{ccccc}
\hline & FT & ET & RT & $\boldsymbol{p}$ \\
\hline Age (Year) & $20.46 \pm 2.29$ & $20.25 \pm 1.85$ & $20.64 \pm 1.94$ & 0.579 \\
Height (Cm) & $166.15 \pm 9.05$ & $164.84 \pm 8.18$ & $165.60 \pm 6.96$ & 0.756 \\
Weight (Kg) & $59.21 \pm 13.10$ & $58.9 \pm 15.20$ & $56.90 \pm 9.27$ & 0.698 \\
\hline
\end{tabular}

FT; flexor taping group

ET; extensor taping group

RT; ring taping group

$* \mathrm{p}<0.05$

\subsection{Changes in Grip and Pinch Power before and after the Application of Taping Treatment}

\subsubsection{Comparison between before and after the Application of Flexor Taping}

Grip power was $30.89 \pm 9.30$ before the application of taping, 32.69 \pm 9.34 after the application of taping in Flexor taping group. Pinch power was $7.12 \pm 1.64$ before the application of taping, and $7.27 \pm 0.56$ after the application of taping. There was a significant change in grip power at the level of 0.00. Although there was also some changes in pinch power, it is not statistically significant $<$ table $2>$.

Table 2. Comparison between before and after of Flexor taping

\begin{tabular}{lcccc}
\hline & Pre-test & Post-test & Change (\%) & $\boldsymbol{p}$ \\
\hline Grip Power & $30.89 \pm 9.30$ & $32.69 \pm 9.34$ & $5.82 \%$ & 0.00 \\
Pinch Power & $7.12 \pm 1.64$ & $7.27 \pm 0.56$ & $2.10 \%$ & 0.37 \\
\hline
\end{tabular}

$* \mathrm{p}<0.05$

\subsubsection{Comparison between before and after the Application of Extensor Taping}

In Extensor taping group, grip power was 33.13 \pm 9.54 before the application of taping, 34.01 \pm 9.39 after the application of taping. Pinch power was $7.66 \pm 1.86$ before the application of taping, 7.70 \pm 2.03 after the application of taping. Both grip power and pinch power showed some improvements after taping. However, there was no statistically significant in Extensor taping group <table $3>$.

Table 3. Comparison between before and after of Extensor taping

\begin{tabular}{lcccc}
\hline & Pre-test & Post-test & Change (\%) & $\boldsymbol{p}$ \\
\hline Grip Power & $33.13 \pm 9.54$ & $34.01 \pm 9.39$ & $2.65 \%$ & 0.12 \\
Pinch Power & $7.66 \pm 1.86$ & $7.70 \pm 2.03$ & $0.52 \%$ & 0.82 \\
\hline
\end{tabular}

${ }^{*} \mathrm{p}<0.05$

\subsubsection{Comparison between before and after the Application of Ring Taping}

In Ring taping group, grip power was $31.21 \pm 8.36$ before the application of ring taping, 32.83 \pm 9.47 after the application of ring taping. Pinch power was $6.95 \pm 1.47$ before the application of ring taping, 7.06 \pm 1.00 after the application of ring taping. Grip power showed significant change at the level of $0.02(p<.05)$. Although there were some changes in pinch power, it was not statistically significant $<$ table $4>$.

Table 4. Comparison between before and after of Ring taping

\begin{tabular}{lcccc}
\hline & Pre-test & Post-test & Change (\%) & $\boldsymbol{p}$ \\
\hline Grip Power & $31.21 \pm 8.64$ & $32.83 \pm 9.47$ & $5.19 \%$ & 0.02 \\
Pinch Power & $6.95 \pm 1.47$ & $7.06 \pm 1.00$ & $1.58 \%$ & 0.67 \\
\hline
\end{tabular}

$* \mathrm{p}<0.05$

\subsection{Comparison of EMG on Application of Taping Treatment in Each Group}

MVC showed statistically significant change at all muscle $(\mathrm{p}<.05)$. According to post-hoc test, Ring taping showed the largest increase in MVC compared with other groups.

Table 5. Comparison of change on EMG in each group.

\begin{tabular}{ccccc}
\hline MVC & FT & ET & RT & $\boldsymbol{p}$ \\
\hline FCU & $10.34 \pm 4.32$ & $9.43 \pm 3.58$ & $15.73 \pm 5.26$ & $0.00^{*}$ \\
FCR & $9.52 \pm 4.87$ & $8.62 \pm 4.63$ & $13.64 \pm 5.48$ & $0.00^{*}$ \\
PL & $10.24 \pm 3.64$ & $10.23 \pm 3.73$ & $15.64 \pm 4.62$ & $0.00^{*}$ \\
\hline
\end{tabular}

FT; flexor taping group

ET; extensor taping group

RT; ring taping group

FCU; flexor carpi ulnaris

FCR; flexor carpi radialis

PL; palmaris longus $* \mathrm{p}<0.05$

\section{Discussion}

Hand consists of 14 phalangeal bones, 5 palmar bones, and 8 carpal bones. The reason for that many bones make up the hand is that the fine joint movement is necessary in this part [18]. Assessment of hand function is commonly performed by measuring the grip strength and pinch strength [11]. Since the function of hand is represented by three movements such as grip, pinch and hook, the coordination of these three movements is the most important factor for the smooth phalangeal motion [19].

Muscle strength changes according to the length of muscle fibers which is one of the mechanical tissues. The maximal tension of muscle increases in the slack condition of muscle fiber and the maximal isometric tension is generated when the sarcomere becomes stable length of

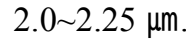

Taping treatment is mainly used for the protection or reinforcement of joints, as well as for decrease of acute injury, pain, and edema in musculoskeletal system. Recently, it is widely used for other purpose such as the 
auxiliary therapy in field of clinics and sports activity [20].

The mechanism of muscle strengthening through the taping therapy is the cutaneous fusimotor reflex. That is, when various stimulus such as contact and vibration stimulates the skin, the action potential is conducted by fusimotor neurons in intrafusal muscles to $\mathrm{Y}$-motor neurons which leads to muscle contraction under skins. As a result, intrafusal muscles contract, and tension of fiber increases, and then both first and second neuroterminal are activated resulting in the improvement of activities in afferent sensory neurons. Thus, muscle strength can be improved after the taping therapy by the increased tension caused from the activation of $\mathrm{y}$-motor neurons in skeletal muscle under the skin [21].

Another mechanism of muscle strengthening of taping therapy is the irradiation phenomenon that intensity of response is increased and spread, once intensity and frequency of stimulation is increased. The increase of contraction force can be resulted from the response of muscle by improving the intensity of stimulation to muscles through taping therapy. Lastly, there is the reciprocal innervation in spinal cord level, arising from sensory receptor of skin [22].

That is, if painful stimulation is given to elbow flexor, $\alpha$-motor neuron in flexor is activated and $\alpha$-motor neuron in extensor is restricted, resulting in facilitation of flexor and inhibition of extensor. Furthermore, crossed extension reflexion that limbs are extended by properly increased stimulation is shown, because one is connected with opposite limbs in the same level of spinal cord by the interneuron.

In other words, the stimulation causing this reflex activates or inhibits the $\alpha$-motor neuron, leading to the contraction or relaxation on flexor and extensor through the synapse. As a result, it compensates the imbalance and helps to maintain the body stability. With this reciprocal innervations phenomenon, the application of taping therapy stimulates the attachment site and activates the contraction of flexor more, inhibits the extensors, which can leads to the improvement of muscle strength [23].

In this study, experiments were conducted with normal adults to investigate the change of grip and pinch power after the application of taping in 3 types such as flexor type, extensor type and ring type. And then, we found outcomes as followings.

Grip power showed the numerical change in all types. However, there is statistical significance in flexor type and ring type, but not significant in extensor type. Pinch power showed no significant change in all 3 types.

This founding that taping therapy is related to muscle strengthening is in line with previous studies. Kim and Jung reported in their study that muscle strength was improved in Y-type taping group after the application of elastic taping on biceps brachii muscle in 2 groups, X-type and Y-type group. Kim et al. also reported that kinesio tape was helpful for the muscle strength and endurance of quadriceps femoris muscle and hamstring muscle during knee flexion and extension [24]. Furthermore, this study is in line with results of Jung and Lee which the muscle strength was improved to maximum during lumbar extension after the taping therapy on erector spinae, quadrates lumborum muscle, rectus abdominis muscle and external oblique muscle.

Lee et al. reported that there was no significant change between before and after taping therapy with elbow band on carpal extensor of normal adults [25]. Lee et al.'s results are partly coherent to this study.

As an aspect of grip power, It is thought that the muscle strength was improved by relaxing antagonist and activating agonist by the taping stimulation after application of flexor and ring type which give the stability to the wrist.

In extensor type, although the muscle strength was expected to improve with the attachment on antagonist, it might affect on stability rather than directly on muscle strengthening. In the same way, pinch power was not significantly changed. It might be because that muscles for pinch were influenced with muscles originating in inner side of hand more than muscle originating medial, lateral epicondyles.

\section{Conclusion}

This study is to investigate the effects of each type, flexor type, extensor type and ring type of taping therapy on the hand grip power and pinch power in normal adults. The results of this study are as followings.

1. After the application of flexor taping, there was a significant change in grip power $(p<0.05)$, but not significant in pinch power $(\mathrm{p}>0.05)$.

2. After the application of extensor taping, both grip power and pinch power showed some increases, but no significance $(\mathrm{p}>0.05)$.

3. After the application of ring taping, there was a significant change $(p<0.05)$, but not statistically significant in the pinch power $(\mathrm{p}>0.05)$.

4. Application of ring taping was significant increase at the MVC compared with other groups $(\mathrm{p}<0.05)$.

Based on these results, flexor and ring type of taping therapy increased the grip power, MVC of hand and is expected to be used as a auxiliary tool for improvement of strength and function in patients who have low hand function because of frequent use of hands. However, in this study, there are some limitations. 1) Difficulty to generalize the results because this study is of healthy normal university students, 2) the fact that Subjects had already known about the taping, 3) the fact that the body condition or psychological condition of the subjects on the day of test and other muscle which can affect the grip or pinch were not considered. If further studies which consider these limitations is conducted, it will contribute to the improvement of muscle strength and function. 


\section{Acknowledgements}

This research was supported by the Korea National University of Transportation Research Grant, 2013.

\section{References}

[1] Thomeé R, Neeter C, Gustavsson A, et al(2012). Variability in leg muscle power and hop performance after anterior cruciate ligament reconstruction. Knee Surgery Sports Traumatology Arthroscopy, 20(6), 1143-1151.

[2] Park SY(1998). A study on the isokinetic muscular strength of shoulder, elbow,knee and trunk in collegiate athletes. Kyung Hee University, Seoul, Korea.

[3] Go HW(1992). Measurement and Evaluation of Sports. Yonsei University, Seoul, Korea.

[4] Marín PJ, Rhea MR(2010). Effects of vibration training on muscle power: a meta-analysis. Journal of Strength and Conditioning Research, 24(3), 871-878.

[5] Sharky BJ(1986). Physiology of Fitness. Human Kinetics.

[6] Kjeken I, Smedslund G, Moe RH, et al(2011). Systematic review of design and effects of splints and exercise programs in hand osteoarthritis. Arthritis Care \& Research, 63(6), 834-848.

[7] Ahn JY, Han JS, Min KS(2000). Experimental study for defining range of motion required for daily activities in wrist joint. The Journal of the Korean Orthopaedic Association, 35(5), 797-802.

[8] González-Iglesias J, Fernández-de-Las- Peñas C, Cleland JA, et al(2009). Short-term effects of cervical kinesio tap-ing on pain and cervical range of motion in patients with acute whiplash injury: a randomized clinical trial. Journal of Orthopaedic and Sports Physical Therapy, 39(7), 515-521.

[9] Chang HY, Wang CH, Chou KY, et al(2012). Could fo-rearm kinesio taping improve strength, force sense, and pain in baseball pitchers with medial epicondylitis? Clinical Journal of Sport Medicine, 22(4), 327-333.

[10] No JK(1998). A Study of the revelation ability of muscles for the increasing flying distance of golfers through the application of the kinesio taping method. Kyonggi University, Seoul, Korea.

[11] Nissenkorn A, Ben-Zeev B(2012). Unilateral rhythmic hand tapping in rett syndrome: is this stereotypy? Journal of Child Neurology, online 21 August.

[12] Chen SM, Alexander R, Lo SK, et al(2012). Effects of Functional Fascial Taping on pain and function in patients with non-specific low back pain: a pilot randomized con-trolled trial. Clinical Rehabilitation, 26(10), 924-933.
[13] Kowall MG, Kolk G, Nuber GW, et al(1996). Patellar taping in the treatment of patellofemoral pain. a prospective randomized study. The American Journal of Sport Medicine, 25(1), 61-66.

[14] Stedge HL, Kroskie RM, Docherty CL(2012). Kinesio taping and the circulation and endurance ratio of the ga-strocnemius muscle. Journal of athletic training, 47(6), 635-642.

[15] Choi KH, Kim HT(2001). The effect of lower leg taping on the muscle performances of ankle joint. The Journal of Korea Society of Physical Therapy, 13(2), 445-452.

[16] Singletary S, Geissler WB(2009). Bracing and rehabilita-tion for wrist and hand injuries in collegiate athletes. Sports Injuries of the Elbow and Hand, 25(3), 443-448.

[17] Jeong CJ, Lee YS(2003). The effect on extension muscle power of waist by taping during exercise. The Korea Journal of Physical Education, 42(6), 849-855.

[18] Kang SH(2000). The contribution of anatomical rotation to racket head velocity on arm segments in a tennis backhand strokes. The Korea Journal of Physical Education, 39(1), 513-519.

[19] Michon M, Maheu E, Berenbaum F(2011). Assessing health-related quality of life in hand osteoarthritis: a litera-ture review. Annals of the Rheumatic Diseases, 70(6), 921-928.

[20] Hubbard TJ, Cordova M(2010). Effect of ankle taping on mechanical laxity in chronic ankle instability. Foot \& Ankle International, 31(6), 499-504.

[21] Johansson H, Sjölander P, Sojka P(1990). Fusimotor ref-lex profiles of individual triceps surae primary muscle spindle afferents assessed with multi-afferent recording technique. Journal de Physiologie 85(1): 6-19.

[22] Hayashi A, Kagamihara Y, Nakajima Y, et al(1988). Dis-order in reciprocal innervation upon initiation of voluntary movement in patients with Parkinson's disease. Experi-mental Brain Research, 70(2), 437-440.

[23] Morgane PJ, Galler JR, Mokler DJ(2005). A review of systems and networks of the limbic forebrain/limbic mid-brain. Progress in Neurobiology, 75(2), 143-160.

[24] Kim MK, Kim BK, Park YJ, et al(2008). The effects of muscle activity and fatigue for vertical jumping on kinesio taping the lower limbs. Journal of Sport and Leisure Studies, 34, 915-923.

[25] Lee YS, Noh JH, Kim CH(2006). Clinical article: effect of elbow band and tapings on normal wrist extensor muscles. Journal of Korean Society of Sports Medicine, 24(2), 237-240. 